\title{
A detector telescope's pitch angle sampling of magnetospheric particles
}

\author{
Miah M. Adel \\ Interdisciplinary Sciences Research Center, Department of Chemistry \& Physics, University of Arkansas at Pine Bluff, Pine Bluff, AR 71601
}

(Received October 5, 2007; Revised January 16, 2008; Accepted March 1, 2008; Online published August 4, 2008)

\begin{abstract}
A geometrical method developed for a particle detector can be extended for application to the determination of the pitch angle sampling capability of the particle telescope of any orientation and for the parent satellite of any orbital orientation. A unit vector tangent to a dipole geomagnetic field line has been calculated from the dipole magnetic field equations in the geomagnetic coordinate system. Rotation matrices have been used, step-by-step, to align the geomagnetic coordinate frame with the detector coordinate frame. The detector selected in this work has its axis tilted with the local zenith direction. The component of the unit vector in the detector coordinate system is found at an observation point characterized by longitude, latitude, and the geocentric radial distance. Pitch angles are measured with respect to the guiding field direction. The pitch angle sampling factor is used in the calculation of detector response functions to particles of different pitch angles. The sampling efficiency functions are useful to make an absolute comparison of magnetospheric particle fluxes measured by particle telescopes under different solar conditions pertaining to different epochs.
\end{abstract}

Key words: Magnetosphere, particle telescope, pitch angle, geomagnetic field, rotation matrix, satellite orbit, efficiency function.

\section{Introduction}

The measurement of anisotropic flux of energetic particles and their pitch angles is indispensable in the study of planetary magnetospheres. Detector telescopes have been flown abroad satellites to detect fluxes of these trapped charged particles (Hovestadt et al., 1972; Moritz, 1972; Miah, 1991a, b; Miah et al., 1992) in the Earth's magnetosphere. The sensitive bases of a cone-shaped instrument detect particles of certain ranges of energy. The size of the opening of the conical detectors samples particles of certain pitch angle range and not particles of a single pitch angle, at a given observation point. The conical detectors are characterized by an opening angle and height. The sensitive base of the detector may be perpendicular to the zenith direction or inclined at some angle from the zenith direction at the observation point. Depending on their geometry and the orientation, particle telescopes onboard have different capabilities of sampling particles of different pitch angles at different latitudes. Detector pitch angle sampling functions (Miah et al., 1989) are useful in comparing particle fluxes measured by particle telescopes under different solar conditions pertaining to different epochs (Miah, 1994).

This article describes how to know the range of the pitch angles detected by the telescopes at any observation point in the orbit. Also, it shows how the relative response func- $\theta$ tion for a given pitch angle $\alpha$ is defined. The detailed calculation is given in another article (Adel, 2007). The orbital information of the satellite, the detector geometry and the detector dimensions used in this work pertain to ONR-

Copyright (c) The Society of Geomagnetism and Earth, Planetary and Space Sciences (SGEPSS); The Seismological Society of Japan; The Volcanological Society of Japan; The Geodetic Society of Japan; The Japanese Society for Planetary Sciences; TERRAPUB.
602 experiment (Miah, 1991a). In the exploration of planetary magnetosphere, knowledge of detector response function is required to calculate the particle flux characterized by anisotropic pitch angle distribution. Simple flux calculation from the detector count rate over a certain time interval using the geometric factor and the energy interval does not consider the anisotropic pitch angle distribution of particles. However, the introduction of the response function considers the anisotropic pitch angle distribution of particles. Incorporation of the detector response functions actually leads to absolute comparison of magnetospheric particle fluxes. The method developed here is very general.

\section{Dynamics of Trapped Particles}

The fundamentals of trapping of charged particles by the geomagnetic field are illustrated in Fig. 1. The guiding field line for a particle performing cyclotron motion between two mirror points near the ends of the magnetic field is shown. Also, the equatorial pitch angle $\alpha_{e}$ at the geomagnetic equator, defined as the angle between the directions of magnetic field $\mathbf{B}_{e}$ at the equator and the velocity $v$ of the particle tangential to its cyclotron orbit is shown in the figure. Further, the geocentric radial distance $r$ and the geocentric equatorial radial distance $r_{o}$, the longitude $\phi$, and the polar angle $\theta$, and the latitude $\lambda$ are illustrated in the figure. The detector in the Earth-orbiting satellite can be located by $(r, \phi, \lambda)$ or $(r, \phi, \theta)$ coordinates.

\section{Setup of Coordinate Axes}

Two coordinate systems are set up-the Earth-centered geomagnetic coordinate system (GCS) $X Y Z$ and the detector coordinate system (DCS) $X^{\prime} Y^{\prime} Z^{\prime}$. In GCS, $X$-axis points northward, $Y$-axis points eastward, and $Z$-axis points vertically downward. In DCS, $Z^{\prime}$-axis points along the tele- 


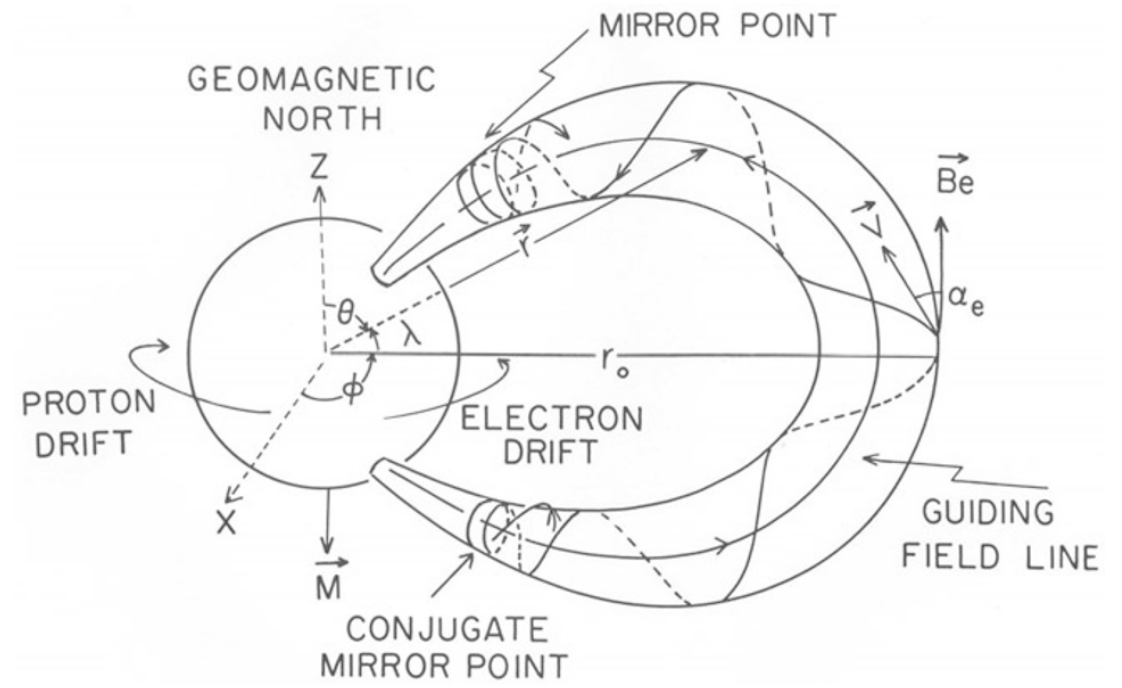

Fig. 1. Particle trapping geometry of the magnetosphere.

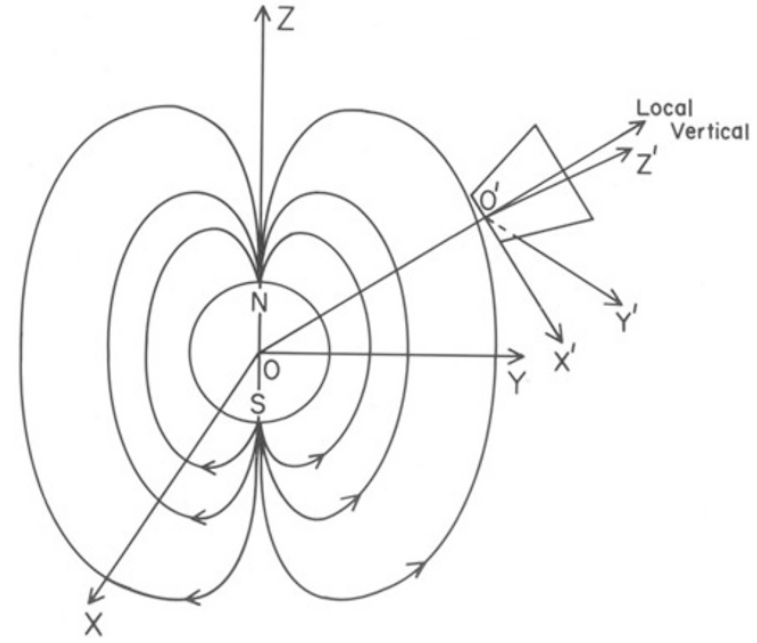

Fig. 2. Coordinate axes for the geomagnetic coordinate system (GCS) $X Y Z$ and the detector coordinate system (DCS) $\mathrm{X}^{\prime} Y^{\prime} Z^{\prime}$.

scope axis which may point along the local vertical direction or be tilted at angle $\delta$ with respect to it and having the direction of tilt to the rightward or leftward of an observer facing the direction of flight. $X^{\prime}$-axis lies in the orbital plane and points opposite to the direction of flight. The orientation of $Y^{\prime}$-axis is chosen to make the coordinate system right-handed. Figure 2 illustrates the coordinate systems.

\section{Unit Tangent Vector along the Magnetic Field} Line in GCS

The equation of a dipole field line is

$$
r=r_{o} \sin ^{2} \theta
$$

An elemental arc length along a field line is given by

$$
\begin{aligned}
d l & =\left[\left(d r^{2}\right)+r^{2}(d \theta)^{2}\right]^{1 / 2} \\
& =r_{o}\left[3 \cos ^{2} \theta+1\right]^{1 / 2} \sin \theta d \theta
\end{aligned}
$$

The radius vector $\mathbf{r}$ is given by

$$
\mathbf{r}=r[\sin \theta \cos \phi \mathbf{i}+\sin \theta \sin \phi \mathbf{j}+\cos \theta \mathbf{k}]
$$

From Eqs. (1) and (3),

$$
\mathbf{r}=r_{o}\left[\sin ^{3} \theta \cos \phi \mathbf{i}+\sin ^{3} \theta \sin \phi \mathbf{j}+\sin ^{2} \theta \cos \theta \mathbf{k}\right]
$$

whence $d \mathbf{r}$ can be written as

$$
\begin{aligned}
d \mathbf{r}= & r_{o}\left[\left(3 \sin ^{2} \theta \cos \theta \cos \phi d \theta-\sin ^{3} \theta \sin \phi d \phi\right) \mathbf{i}\right. \\
& +\left(3 \sin ^{2} \theta \cos \theta \sin \phi d \theta+\sin ^{3} \theta \cos \phi d \phi\right) \mathbf{j} \\
& \left.+\left(2 \sin \theta \cos ^{2} \theta-\sin ^{3} \theta\right) d \theta\right] \mathbf{k}
\end{aligned}
$$

The unit tangent vector at $(r, \theta, \phi)$ is defined as

$$
\mathbf{t}=d \mathbf{r} / d l
$$

since

$$
d \phi / d \theta=0
$$

From Eqs. (2) and (5)

$$
\begin{aligned}
\mathbf{t}= & {[3 \sin \theta \cos \theta \cos \phi \mathbf{i}+3 \sin \theta \cos \theta \sin \phi \mathbf{j}} \\
& \left.+\left(2 \cos ^{2} \theta-\sin ^{2} \theta\right) \mathbf{k}\right] /\left[3 \cos ^{2} \theta+1\right]^{1 / 2} \\
= & U \mathbf{i}+V \mathbf{j}+W \mathbf{k}
\end{aligned}
$$

The above formulations show that $\mathbf{t}$ is independent of $\mathbf{r}$.

\section{Rotation of $t$}

A series of rotation matrices are applied to align the GCS with the DCS. The objective is to get the components of $\mathbf{t}$ in DCS. In GCS, we apply the first rotation around $O Z$

(1) axis through the longitude $\phi$ of observation in the anticlockwise direction. This rotation brings $Z X$-plane along the magnetic meridian at the observation point. The rotation matrix is

$$
T^{\prime}=\left(\begin{array}{ccc}
\cos \phi & \sin \phi & 0 \\
-\sin \phi & \cos \phi & 1 \\
0 & 0 & 1
\end{array}\right)
$$


In the rotated frame $\mathbf{t}$ appears as

$$
\mathbf{t}^{\prime}=U^{\prime} \mathbf{i}+V^{\prime} \mathbf{j}+W^{\prime} \mathbf{k}
$$

where

$$
\begin{aligned}
U^{\prime} & =U \cos \phi+V \sin \phi \\
V^{\prime} & =-U \sin \phi+V \cos \phi \\
W^{\prime} & =W
\end{aligned}
$$

If expressions for $U$ and $V$ are substituted in Eq. (12), it is found that $U^{\prime}$ and $V^{\prime}$ become independent of longitude. This is where the longitude term drops out. Thus the calculated response function will be independent of $r$ and $\phi$ in a dipole magnetic field.

The next rotation is around $O Y$ axis in the anti-clockwise sense through the polar angle $\theta$ to bring the $O Z$ axis along the local zenith direction at the latitude $\lambda$ of observation, where

$$
\lambda=90^{\circ}-\theta
$$

The rotation matrix is

$$
\begin{aligned}
T^{\prime \prime} & =\left(\begin{array}{ccc}
\cos \theta & 0 & -\sin \theta \\
0 & 1 & 0 \\
\sin \theta & 0 & \cos \theta
\end{array}\right) \\
& =\left(\begin{array}{ccc}
\sin \lambda & 0 & -\cos \lambda \\
0 & 1 & 0 \\
\cos \lambda & 0 & \sin \lambda
\end{array}\right)
\end{aligned}
$$

This leads to

$$
\mathbf{t}^{\prime \prime}=U^{\prime \prime} \mathbf{i}+V^{\prime \prime} \mathbf{j}+W^{\prime \prime} \mathbf{k}
$$

where

$$
\begin{aligned}
U^{\prime \prime} & =U^{\prime} \sin \lambda-W^{\prime} \cos \lambda \\
V^{\prime \prime} & =V^{\prime} \\
W^{\prime \prime} & =U^{\prime} \cos \lambda+W^{\prime} \sin \lambda
\end{aligned}
$$

The above two rotations leave the $O Z$ axis in the orbital plane, $Z X$ aligned with the longitude $\phi$, and so perpendicular the geomagnetic equator.

The third rotation is along the new $O Z$ axis in the clockwise sense through such an angle as to align the rotated $Z X$-plane with the satellite orbit. This angle is a function of both the satellite's orbital inclination $\psi$ with the equatorial plane and the geomagnetic latitude of observation, $\lambda$. The geomagnetic latitude can be evaluated using spherical trigonometry illustrated in Fig. 3. We use the properties of right spherical triangles to estimate the required rotation $\eta$. OPQ is the right spherical triangles. In the figure, the lower case letters represent the angles subtended at the geo-center by the sides they designate. The upper case letters represent the angles of the triangle on the spherical surface. The angle $\mathrm{Q}$ is $90^{\circ}$, because it is the point of intersection of a meridian with the equator. $\mathrm{OQ}=p$ subtends at the geo-center the orbital inclination angle i.e., $\mathrm{OQ}=\psi$. The satellite's orbit is along PO, and PQ is part of the equator. Angle $p$ is given by the relation

$$
\sin o=\tan p \cot \mathrm{P}
$$

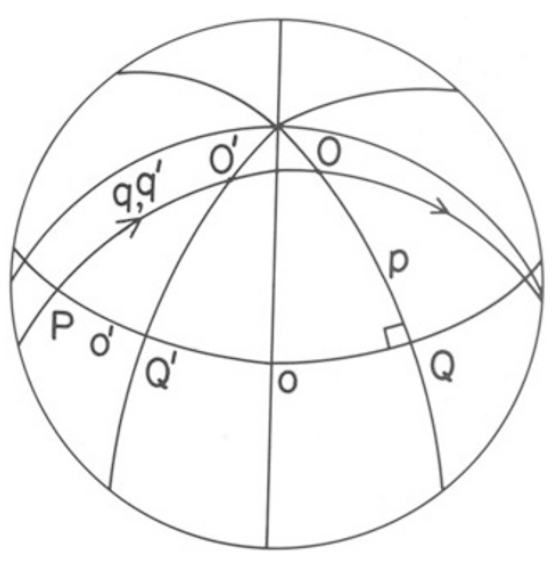

Fig. 3. Illustration for the determination of the angle $\eta$ rotation through which aligns the $Z X$-plane with satellite orbit. The line with arrows indicates the satellite orbit.

where $o=\mathrm{PQ}=90^{\circ}$ on the grounds that if $\mathrm{O}$ is the highest point of ascension, OQ equally divides the satellite's orbit (assumed circular) in the northern half of the globe. The angle $p$ as given by the above relation is equal to the angle of orbital inclination. Now, let $\mathrm{O}^{\prime}$ be the point where the satellite is located. $\mathrm{O}^{\prime} \mathrm{PQ}^{\prime}$ is again a right spherical triangle. $\mathrm{O}^{\prime} \mathrm{Q}^{\prime}$ is the longitude at the observation point. $\mathrm{O}^{\prime} \mathrm{Q}^{\prime}$ is then $p$. We now use the following relation

$$
\cos p=\cos p^{\prime} \sin \mathrm{O}^{\prime}
$$

to obtain the angle $\mathrm{O}^{\prime}$ at the observation point. Here angle $\mathrm{O}^{\prime}$ is $\eta$, the angle through which $Z X$ plane has to be rotated in the clockwise direction to align with the orbital plane. The rotation matrix for this is:

$$
T^{\prime \prime \prime \prime}=\left(\begin{array}{ccc}
\cos \eta & -\sin \eta & 0 \\
\sin \eta & \cos \eta & 0 \\
0 & 0 & 1
\end{array}\right)
$$

and the rotated unit vector is

$$
\mathbf{t}^{\prime \prime \prime \prime}=U^{\prime \prime \prime} \mathbf{i}+V^{\prime \prime \prime} \mathbf{j}+W^{\prime \prime \prime} \mathbf{k}
$$

where

$$
\begin{aligned}
U^{\prime \prime \prime} & =U^{\prime \prime} \cos \eta-V^{\prime \prime} \sin \eta \\
V^{\prime \prime \prime} & =U^{\prime \prime} \sin \eta+V^{\prime \prime} \cos \eta \\
W^{\prime \prime \prime} & =W^{\prime \prime}
\end{aligned}
$$

If $\psi=90^{\circ}$, then the angle $\eta$ obtained above is to be applied for a satellite traveling northward. For the southward trip, $\eta$ has to be replaced by $180^{\circ}-\eta$. For $\phi>90^{\circ}, \eta=\mathrm{O}^{\prime}$ is a negative angle in Eq. (19), and in that case the above matrix denotes rotation in the anticlockwise direction through the angle $\eta$ for flight direction to the north, and through $180^{\circ}-\eta$ for flight direction to the south.

After the third rotation, the $Z X$-plane is aligned with the orbital plane, $O Z$-axis points along the local zenith, and $Y Z$ plane is normal to the orbital plane.

The last rotation for alignment of coordinate systems is through the angle of tilt, $\delta=2.35^{\circ}$ in the clockwise sense. The axis of the telescope is tilted with the local 
zenith direction. The direction of tilt is to the right of an observer moving in the direction of flight. We can think of the situation in the following way: The rotated GCS has its $O Z$ axis pointing in the vertical direction while it lies in the plane of the satellite's orbit. The DCS $O^{\prime} Z^{\prime}$ axis lies in a plane perpendicular to the orbital plane and is inclined in the clockwise direction from the local vertical by an angle $\delta\left(=2.35^{\circ}\right)$ The rotation matrix to be used is the one for clockwise rotation around $O X$ axis, which is

$$
T^{\prime \prime \prime \prime}=\left(\begin{array}{ccc}
1 & 0 & 0 \\
0 & \cos \delta & -\sin \delta \\
0 & \sin \delta & \cos \delta
\end{array}\right)
$$

The rotated unit vector is

$$
\mathbf{t}^{\prime \prime \prime \prime}=U^{\prime \prime \prime \prime} \mathbf{i}+V^{\prime \prime \prime \prime} \mathbf{j}+W^{\prime \prime \prime \prime} \mathbf{k}
$$

where

$$
\begin{aligned}
U^{\prime \prime \prime \prime} & =U^{\prime \prime \prime} \\
V^{\prime \prime \prime \prime} & =V^{\prime \prime \prime} \cos \delta-W^{\prime \prime \prime} \sin \delta \\
W^{\prime \prime \prime \prime} & =V^{\prime \prime \prime} \sin \delta+W^{\prime \prime \prime \prime} \cos \delta
\end{aligned}
$$

\section{Rotation to Cover the Finite Detector Area}

Detector base has a finite area. Figure 4 illustrates the detector. What has been done above is applicable for the central point on the detector base that defines the full opening angle. To cover the entire area of the detector, the detector area is divided into concentric rings and each annular area into segments of equal areas, the central points of which are taken as the representative points to cover the entire base area. Figure 5 illustrates the division into segments. The area of the $n$th annular ring is

$$
(2 n+1) \pi r^{2}
$$

where

$$
r=\frac{R}{N}
$$

and

$$
\begin{aligned}
R & =\text { radius of the detector } \\
N & =\text { number of annular rings } \\
& =\text { number of divisions in the radius }
\end{aligned}
$$

The radial coordinate of the middle point of the $n$th section is given by

$$
r_{n}=(2 n+1) r / 2
$$

where $n$ represents the number of the annular rings, the central ring having $n=0$. And the angular coordinate, by

$$
\gamma_{n}=\left(2 n^{\prime}+1\right) / 2.360 /(2 n+1)
$$

for the $n^{\prime}$ th area in the $n$th ring $(n \geq 1)$. $n^{\prime}$ represents the segment number in the $n$th ring, $n^{\prime}$ being equal to 0 for the first and $2 n$ for the last segment.

The final rotated unit tangent vector is obtained from anticlockwise vector rotation through $\gamma$ around the $O Z$ axis. Its components are

$$
\begin{aligned}
& B_{x}=U^{\prime \prime \prime \prime} \cos \gamma-V^{\prime \prime \prime \prime} \sin \gamma \\
& B_{y}=U^{\prime \prime \prime \prime} \sin \gamma+V^{\prime \prime \prime \prime} \cos \gamma \\
& B_{z}=V^{\prime \prime \prime \prime} .
\end{aligned}
$$

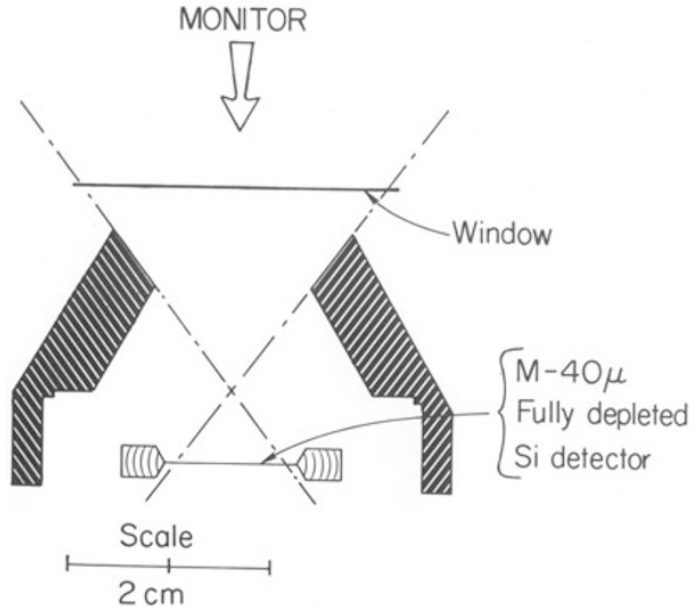

Fig. 4. The particle telescope used in this work.

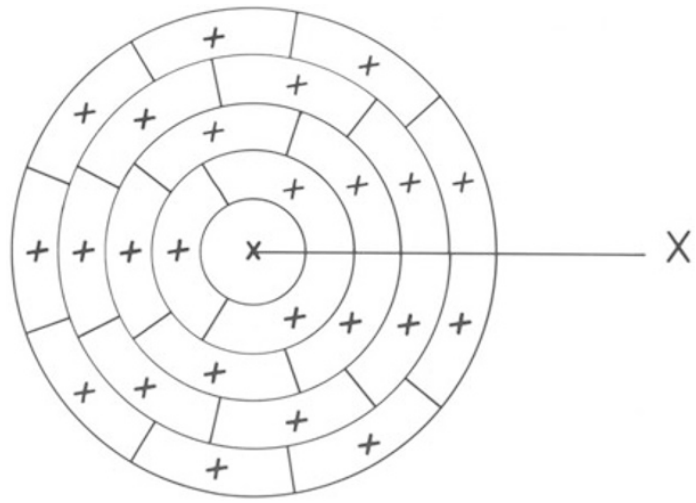

Fig. 5. Division of the detector sensitive base into elemental areas $\Delta A$.
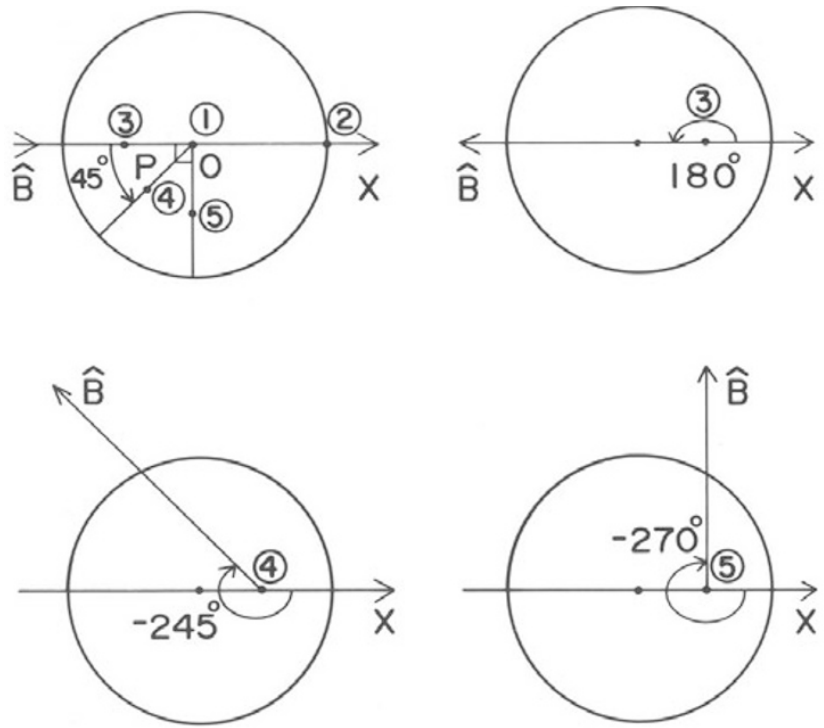

Fig. 6. Illustration of the additional rotations required when the detector telescope apex lies at an off-centered point on the sensitive base.

The detector telescope is a right circular cone only for the central point on the detector base. For those off-centered points above into which the telescope base is divided for full coverage, the detector telescope forms only circular cones. 
If the off-centered points fall on the positive $x$-axis, only translation of the unit vector is involved. Points that do not fall on the $x$-axis have non-zero $\gamma$ values. To reach these points, we have to do translation of the unit vector along the $X$-axis followed by rotations through angles of $-180^{\circ}$, $-245^{\circ},-270^{\circ}$, and so on (Fig. 6).

The unit tangent of the magnetic vector is given as a function of geomagnetic latitude, longitude, orbital plane inclination with the equatorial plane, direction of flight, and the tilt angle of the telescope axis with the local vertical direction. We need to know the unit vector along the incident direction of the incoming particle. A dot product of the two unit vectors will give the pitch angle of the incoming particle.

\section{Unit Vector along the Particle Incident Direc- tion}

For convenience, contrary to the actual case, incident vectors are taken positive opposite to the incident direction i.e. in the upward direction. This does not change the sampling efficiency at all. How the coordinates of the incident vectors are found is discussed below.

We project the magnetic vector onto the opening ring of the telescope. In Fig. 7, the chord $\mathrm{PO}^{\prime} \mathrm{Q}$ represents the projection. The angle $\chi$ between the positive $X$-axis and $\mathrm{PO}^{\prime} \mathrm{Q}$ is given by

$$
\chi=\cos ^{-1}\left(\frac{B_{x}}{\sqrt{\left(B_{x}^{2}+B_{y}^{2}\right)}}\right)
$$

If $B_{y}$ is negative, $\chi$ has to be subtracted from $360^{\circ}$ to get the actual value of $\chi$. When the origin of the coordinate frame is at $(a, 0)$, the coordinate $X$ is given by

$$
X=R \cos \left(\chi^{\prime}\right)-a
$$

where $\chi^{\prime}$ is obtained from the law of cosines of the triangle $\mathrm{POO}^{\prime}$ formed with the point $\mathrm{P}$ of incidence on the telescope opening ring, the center $\mathrm{O}$ of the opening ring, and the projection $\mathrm{O}^{\prime}$ on the opening ring of the point under consideration in the detector base. In triangle $\mathrm{POO}^{\prime}$,

$$
\text { angle } \mathrm{PO}^{\prime} \mathrm{O}=180^{\circ}-\chi
$$

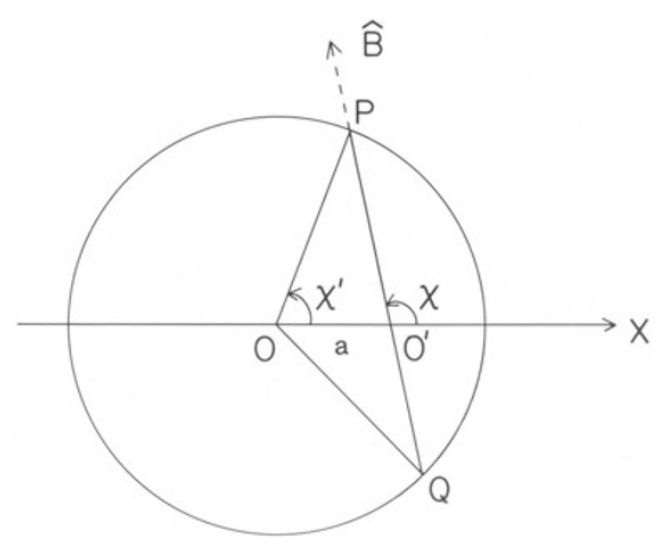

Fig. 7. Illustration of the angle $\chi$ and $\chi^{\prime}$, being the angle between the $X$-axis and the projection of the magnetic field on the detector opening ring.
OP is the radius $R$ of the opening ring. $\mathrm{OO}^{\prime}$ is equal to $a$, the shift of the origin along the $X$ axis. The length of the chord $\mathrm{PO}^{\prime}$ is obtained from

$$
R^{2}=\mathrm{PO}^{\prime 2}+a^{2}-2 a \mathrm{PO}^{\prime} \cos \left(180^{\circ}-\chi\right)
$$

The knowledge of the length $\mathrm{PO}^{\prime}$ lets us calculate the angle $\mathrm{POO}^{\prime}$ which is $\chi^{\prime}$. Using the relation

$$
\mathrm{PO}^{\prime 2}=R^{2}+a^{2}-2 a R \cos \chi^{\prime}
$$

The $Y$ coordinate is given by

$$
Y=R \sin \chi^{\prime}
$$

The $Z$ coordinate is the height of the telescope cone. The telescope under consideration has

$$
Z=H=1.7336 \mathrm{~cm}
$$

The unit vector for a point on the opening ring of the telescope is found from the $X, Y$, and $Z$ coordinates. The unit vector for this point and the unit magnetic field vector determine the pitch angle of the incoming particles. The cosine of the pitch angle is given by

$$
\cos \alpha=B_{x} \cdot X^{\prime}+B_{y} \cdot Y^{\prime}+B_{z} \cdot Z^{\prime}
$$

where $X^{\prime}, Y^{\prime}$, and $Z^{\prime}$ are the direction cosines of the incident unit vector.

\section{Limits of Pitch Angles}

The minimum and maximum pitch angles of particles which can enter the detector are found referring to Fig. 8. The angle $\chi^{\prime \prime \prime}$ lies between the magnetic field vector and the instrument $Z$-axis. The minimum pitch angle is

$$
\alpha_{\min }=\chi^{\prime \prime \prime}-\alpha_{1}
$$

The maximum pitch angle is

$$
\alpha_{\max }=\chi^{\prime \prime \prime}+\alpha_{2}
$$

The angles $\alpha_{1}$ and $\alpha_{2}$ are calculated from lengths $\mathrm{CD}$ (which is $\mathrm{O}^{\prime} \mathrm{P}$ in Fig. 7), DA (which is $\mathrm{O}^{\prime} \mathrm{Q}$ in Fig. 7) and $\mathrm{OD}$ (which is the telescope height). The extreme pitch angles can also be calculated from the unit magnetic field vector and the unit vectors directed from the apex of telescope cone to points $\mathrm{P}$ and $\mathrm{Q}$. The two ways yield the same result.

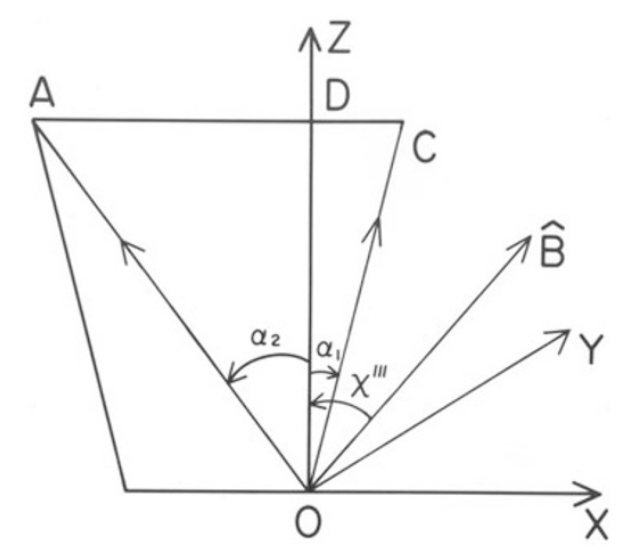

Fig. 8. Illustration to determine the minimum and maximum pitch angle for an off-centered position of the detector telescope apex. 


\section{Latitude Dependence of Extreme Pitch Angles}

The above expressions of $\alpha_{\min }$ and $\alpha_{\max }$ put some limitations in the latitude range on the validity of this method of response function calculation. In a latitude at which the angle between the magnetic field and the $Z$-axis $\chi^{\prime \prime \prime}$ is less than $\alpha_{1}$ then $\alpha_{\min }=0^{\circ}$, and

$$
\alpha_{\max }=\chi^{\prime \prime \prime}+\alpha_{2}
$$

The maximum values of $\alpha_{1}$ is

$$
\alpha_{1}=\alpha_{2}=24.11^{\circ} \text { for } a=0
$$

The minimum values of $\alpha_{1}$ is $\approx 7^{\circ}$ for $a=0.56388 \mathrm{~cm}$. The maximum value of $\alpha_{2}$ is then $37.7^{\circ}$. At $+30^{\circ}$ latitude, $\chi^{\prime \prime \prime}=41^{\circ}$ and at $-30^{\circ}$ latitude $\chi^{\prime \prime \prime}=139^{\circ}$. So, within this latitude range, the calculated efficiency function is quite valid.

\section{Precise Determination of Extreme Pitch An- gles}

The fact that the pitch angle cone is always right circular and the telescope cone is right circular only when its apex lies at the central point of the detector (the telescope cone for an off-centered incidence of the particle on the detector sensitive base is not a right circular cone) needs to be considered in the precise determination of the minimum and maximum pitch angles. The intersection of the pitch angle cone and the plane of the opening ring of the telescope is a conic section having the projection of the magnetic field vector on the opening ring of the telescope as the axis of symmetry. Figure 9 illustrates that for a given magnetic field position, calculation takes the point $\mathrm{P}$ as the point of contact to determine the minimum pitch angle cone, whereas it should be $\mathrm{P}^{\prime}$. To get to $\mathrm{P}^{\prime}$ as closely as possible, $\chi^{\prime}$ is steadily increased by a fraction of a degree, the calculated pitch angle first increases and then decreases. The value of $\chi^{\prime}$ at the turning point corresponds to the minimum pitch angle. The same way, the maximum pitch angle can be more precisely located.

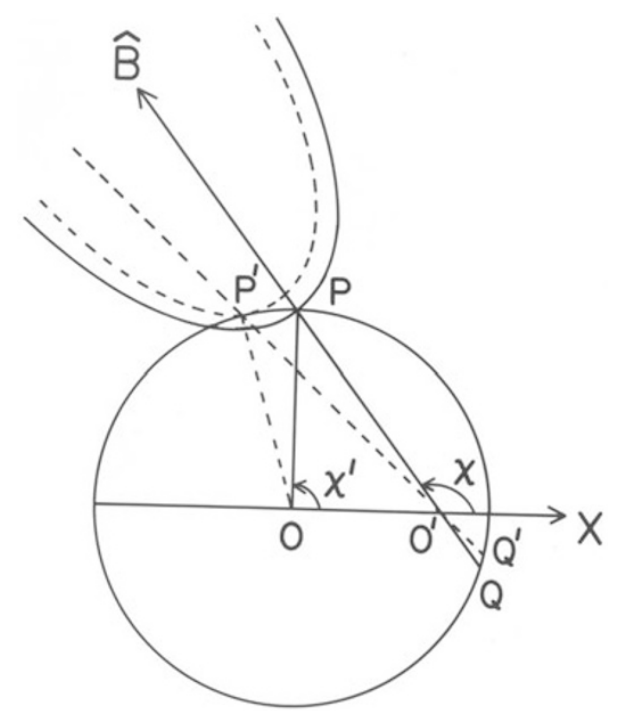

Fig. 9. Illustrates the exact determination of the minimum and maximum pitch angle for the telescope cone.

\section{Iterative Process to Solve the Pitch Angle}

First, a given pitch angle within the instrumental pitch angle range is taken. Then the two points of intersections of the pitch angle cone with the telescope cone are found by iteration so that the difference between the given pitch angle and the computed pitch angle is less than some small number (for example, $0.001^{\circ}$ ). In the iteration process, the two points of intersection are sought on either side of the projection of the magnetic field vector on the opening ring of the telescope. Figure 10 illustrates the iterative procedure.

$$
\begin{aligned}
& \text { Angle } \mathrm{P}^{\prime} \mathrm{O}^{\prime} \mathrm{P}=\chi \\
& \text { Angle } \mathrm{P}^{\prime} \mathrm{OO}^{\prime}=\chi^{\prime} \\
& \text { Angle } \mathrm{P}^{\prime} \mathrm{OP}^{\prime \prime}=\Delta \chi^{\prime}
\end{aligned}
$$

and

$$
\text { angle } \mathrm{P}^{\prime} \mathrm{O}^{\prime} \mathrm{P}^{\prime \prime}=\Delta \alpha
$$

Regarding the coordinate frame, $X Y$-plane is the plane of the detector, and $\mathrm{O}^{\prime \prime} \mathrm{O}^{\prime}$ points along the $Z$-axis (both $Z$-and $Y$-axes are not shown in the figure).

The iteration step size is

$$
\Delta \chi^{\prime} \approx \frac{l \Delta \alpha}{R+a}
$$

where $l\left(=\mathrm{O}^{\prime \prime} \mathrm{P}\right)$ is the slant height of the telescope cone which cuts the telescope ring at $X=R-a, Y=0$, and $Z=1.7336 \mathrm{~cm}$. The approximate relation is obtained from the expressions for elemental changes, in $\chi^{\prime}$ defined in terms of polar variable $r$ on the opening ring of the telescope cone:

$$
\Delta \chi^{\prime}=\frac{\Delta r}{r}
$$

and the pitch angle is defined in terms of the slant height $l$ of the telescope cone

$$
\Delta \alpha \approx \frac{\Delta l}{l}
$$

In the approximate relation

$$
\Delta \chi^{\prime} \approx \frac{l \Delta \alpha}{R+a}
$$

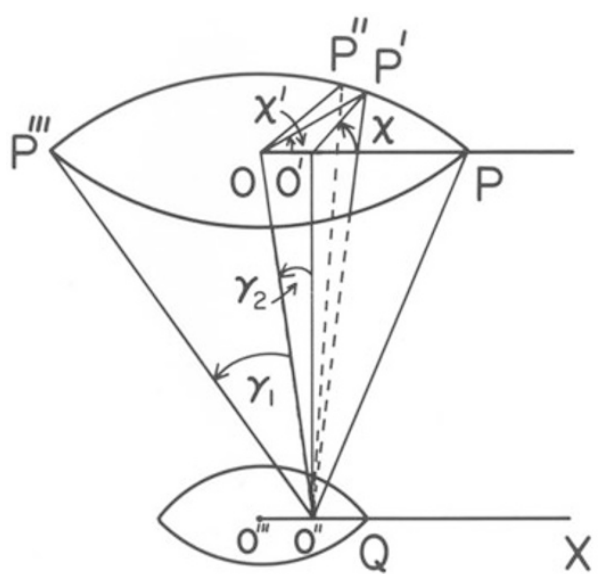

Fig. 10. Illustrates the formulation of the general form of the equation for the telescope cone. 
The least value of

$$
l=\left(H^{2}+(R-a)^{2}\right)^{\frac{1}{2}}
$$

and the largest value of

$$
r=R+a
$$

have been made. $r$ may also be given the constant value $R$. This keeps $\Delta \chi$ as small as possible and makes $\Delta \alpha$ slowly converge toward zero. Each time $\Delta \chi$ is added to $\chi, X$ and $Y$ coordinates are determined. The unit vector along the incident direction is calculated from $X, Y$, and $Z$ coordinates. A dot product of this vector with the unit vector of the magnetic field $\mathbf{B}$ gives the pitch angle for the point under consideration. The $\Delta \alpha$ is calculated, and the above steps are repeated until $\Delta \alpha$ falls lower than 0.001 . The entire operation is repeated on the other side of the $\mathbf{B}$ vector position, and the second point of intersection is found for the pitch angle under consideration.

\section{Accuracy of the Solution of the Pitch Angle Equation}

A check was made to see if the two points of intersection satisfy both the pitch angle and the telescope cone equations. The following equation for the telescope was deduced for the cone at the apex $(a, 0,0)$ :

$$
\left(X+H \tan \gamma_{1}\right)^{2}+Y^{2}=H^{2}\left(\tan \left(\gamma_{1}+\gamma_{2}\right)-\tan \gamma_{1}\right)^{2}
$$

In Fig. $10, \mathrm{O}^{\prime \prime} \mathrm{O}^{\prime}$ is the cone axis angle $\mathrm{O}^{\prime} \mathrm{O}^{\prime \prime} \mathrm{O}=\gamma_{2}$ and the angle $\mathrm{OO}^{\prime \prime} \mathrm{P}^{\prime \prime \prime}=\cdot \gamma_{1}$

This can be simplified to

$$
X^{2}+Y^{2}+2 a X-2(R+a)(R-a)=0
$$

Where in the figure, $a=\mathrm{OO}^{\prime}\left(=\mathrm{O}^{\prime \prime \prime} \mathrm{O}^{\prime \prime}\right)$, the distance from the central point of the detector to the telescope cone, and $\mathrm{OP}=R$, the radius of the opening ring. The pitch angle cone is written with the magnetic field vector as the axis. Later, through transformation of coordinates, the equation is obtained in the telescope cone coordinate system. This equation is

$$
Y^{\prime \prime \prime 2}+Z^{\prime \prime \prime 2}=X^{\prime \prime 2} \tan ^{2} \alpha
$$

Where $\alpha$ is the given pitch angle. The first rotation is in the clockwise direction around $O Z$-axis through $\chi$ deduced from Eq. (31). This yields

$$
\begin{aligned}
& X^{\prime}=X \cos \chi+Y \sin \chi \\
& Y^{\prime}=-X \sin \chi+Y \cos \chi \\
& Z^{\prime}=Z
\end{aligned}
$$

The next rotation is around $O Y^{\prime}$ axis through angle $\chi^{\prime \prime}=$ $90^{\circ}-\chi^{\prime \prime \prime}$, the angle between the magnetic vector $\mathbf{B}$ and the $Z$-axis unit vector $\mathbf{k}$ in the anticlockwise direction. The rotaltion yields:

$$
\begin{aligned}
& X^{\prime \prime}=X^{\prime} \cos \chi^{\prime \prime}+Z^{\prime} \sin \chi^{\prime \prime} \\
& Y^{\prime \prime}=Y^{\prime} \\
& Z^{\prime \prime}=-X^{\prime} \sin \chi^{\prime \prime}+Z^{\prime} \cos \chi^{\prime \prime}
\end{aligned}
$$

The above three equations define the pitch angle cone in the telescope cone frame.

Substitution of the coordinates of the two points of intersection found by iteration satisfies the telescope cone equation exactly. However, the pitch angle equation yields a value of about $1 \times 10^{-4}$ for pitch angles not equal to $90^{\circ}$, and for pitch angle equal $90^{\circ}, \chi^{\prime \prime 2}$ yields about $1 \times 10^{-6}$. The accuracy of the solution depends upon the condition that the difference between the given and computer pitch angles should be less than $0.001^{\circ}$.

\section{Sampling Efficiency}

The fractional omnidirectional efficiency $f(\alpha)$ for a particle of specific pitch angle $\alpha$ is the fraction of the associated space angle intercepted by the telescope cone duly weighted by the perpendicular component of the fractional detector area exposed to the incoming particles, and normalized by the efficiency of a half-omnidirectional detector. Figure 11 illustrates the fractional efficiency determination which is embedded in the integral.

$$
G=\int_{\alpha_{1}(d A)}^{\alpha_{2}(d A)} d \alpha \int_{d \Omega} d \omega \int_{A} d \mathbf{A} \cdot \mathbf{r}(\omega) \cdot \sin ^{q} \alpha
$$

where $d \Omega$ is the domain of the solid angle $\omega$ and is determined from the pitch angle cone and the telescope cone geometry, $d \omega=\sin \alpha d \beta^{\prime \prime}, \alpha$ being the polar angle and $\beta^{\prime \prime}$ the azimuth angle. The perpendicular component of the area element is $d \mathbf{A} . \mathbf{r}(\omega)$ looking into $\omega$. It may be understood that $G$ is a product of the detector sensitive area perpendicular to the incoming particle beam, an effective solid angle, and the pitch angle distribution. Its unit is $\mathrm{cm}^{2}$.sr.

$G=\int_{\alpha_{1}(d A)}^{\alpha_{2}(d A)} \sin ^{q} \alpha d \alpha \int_{\beta_{\min }^{\prime \prime}\left(\alpha, d A, \chi^{\prime \prime \prime}\right)}^{\beta_{\max }^{\prime \prime}\left(\alpha, d A, \chi^{\prime \prime \prime}\right)} \sin \alpha d \beta^{\prime \prime} \int_{A} d \mathbf{A} \cdot \mathbf{r}(\omega)$

With $\mathbf{r}$ and $d \mathbf{A}$ given by

$$
\mathbf{r}=\sin \alpha \cos \beta^{\prime \prime} \mathbf{i}+\sin \alpha \sin \beta^{\prime \prime} \mathbf{j}+\cos \alpha \mathbf{k}
$$

and

$$
\begin{aligned}
d \mathbf{A}= & \sin \chi^{\prime \prime \prime} \mathbf{i}+0 \mathbf{j}+\cos \chi^{\prime \prime \prime} \mathbf{k} \\
\int_{A} d \mathbf{A} \cdot \mathbf{r}(\omega)= & \int_{A} d A\left(\sin \alpha \cos \beta^{\prime \prime} \sin \chi^{\prime \prime \prime}\right. \\
& \left.+\cos \alpha \cos \chi^{\prime \prime \prime}\right) \\
\approx & \sum_{i=1}^{n} \Delta A_{i}\left(\sin \alpha_{i} \cos \beta^{\prime \prime} \sin \Delta \chi^{\prime \prime \prime}\right. \\
& \left.+\cos \alpha_{i} \cos \chi^{\prime \prime \prime}\right)
\end{aligned}
$$

The entire integration is then

$$
\begin{aligned}
G= & A \cdot \sum_{j=1}^{m} \sin ^{q}\left(\alpha_{j}\right) \Delta \alpha \cdot \sum_{i=1}^{n} \frac{\Delta A_{i}}{A}\left[\sin ^{2} \alpha_{i j} \sin \chi^{\prime \prime \prime}\right. \\
& \left.\cdot\left(\sin \beta_{\max }^{\prime \prime}-\sin \beta_{\min }^{\prime \prime}\right)+\sin \alpha_{i j} \cos \alpha_{i j} \cos \chi^{\prime \prime \prime}\right) \\
& \left.\cdot\left(\sin \beta_{\max }^{\prime \prime}-\sin \beta_{\min }^{\prime \prime}\right)\right]
\end{aligned}
$$

where $\Delta \alpha=\left(\alpha_{2}-\alpha_{1}\right) / m$ is in radian units

$$
=A \cdot \sum_{j=1}^{m} F\left(\alpha_{j}\right) \Delta \alpha f\left(\alpha_{j}\right)
$$




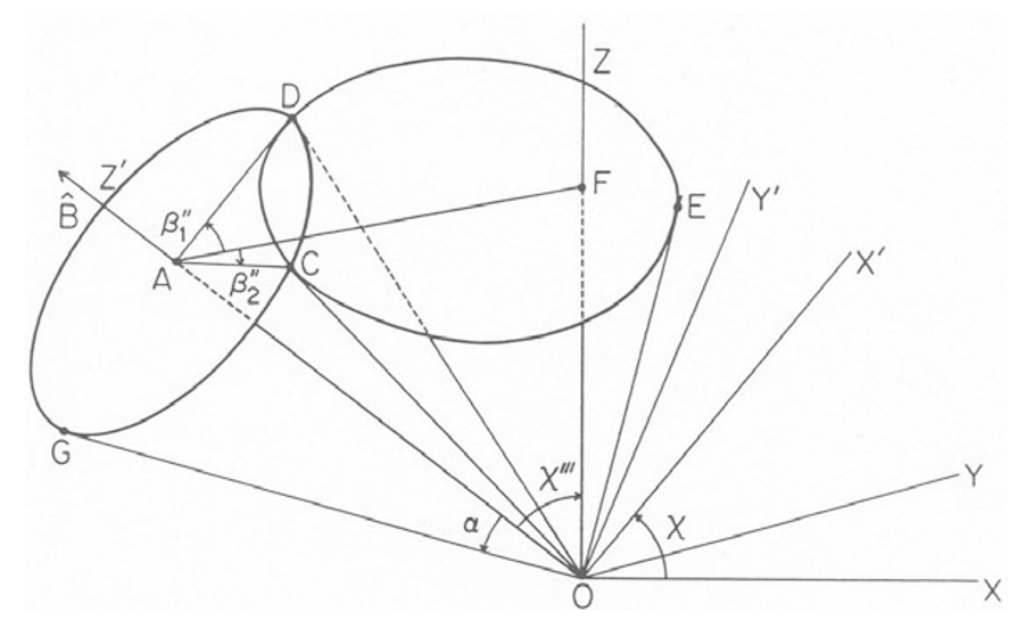

Fig. 11. Illustrates the fractional associated space angle intercepted by the telescope cone.

where $F\left(\alpha_{j}\right)=\sin ^{q} \alpha_{j}$, the pitch angle distribution function, and

$$
\begin{aligned}
f\left(\alpha_{j}\right)= & \frac{1}{2 \pi} \sum_{i=1}^{n} \frac{\Delta A_{i}}{A}\left[\sin ^{2} \alpha_{i j} \sin \chi^{\prime \prime \prime}\left(\sin \beta_{\max }^{\prime \prime}-\sin \beta_{\min }^{\prime \prime}\right)\right. \\
& \left.+\sin \alpha_{i j} \cos \alpha_{i j} \cos \chi^{\prime \prime \prime}\right) \\
& \left..\left(\sin \beta_{\max }^{\prime \prime}-\sin \beta_{\min }^{\prime \prime}\right)\right]
\end{aligned}
$$

is the efficiency function for $\alpha_{j}$ applied to the whole detector. At the beginning, we select a pitch angle. We start with the central point of the detector. This gives us $f_{1}$. We then calculate $f_{i}$ at all the representative points. We then sum and find the average according to

$$
f=\sum \frac{f_{i}}{(N+1)^{2}}
$$

\section{Coverage, Magnetic Field-induced Variabil- ity, and Application to Measured Data}

As to the area of applicability, the method is applicable at any latitude and longitude. To show it qualitatively, we can pick up only the equatorial region for the equatorially mirroring particles for which we know the pitch angle $\left(\alpha_{e}\right)$ distribution function. It has the form $\sin ^{n} \alpha_{e}$ where $n$ runs from 5 to 21 from different observation with the most likely value in the middle of the range (Miah, 1988). Sampling function at the equator can be approximated by the same sine function with exponent 13. So the detector can respond to all the equatorially mirroring particles. For off-equatorially mirroring particles at the equator, it cannot detect any particles beyond the equatorial pitch angle $90^{\circ} \pm 37^{\circ}$.

If we know the pitch angle distribution function of particles mirroring at other latitudes, we can check with the applicability of the method with the instrument response function calculated at that latitude.

As to the influence by the activity of the Earth's magnetosphere, the response function calculated depends upon the magnetic field orientation. Magnetospheric activities are influenced by the solar conditions. The response function thus depends on the solar conditions. Whereas no calculations were made for solar quiet and violent conditions, some differences were observed between the response functions calculated for the dipole field and the real geomagnetic field.

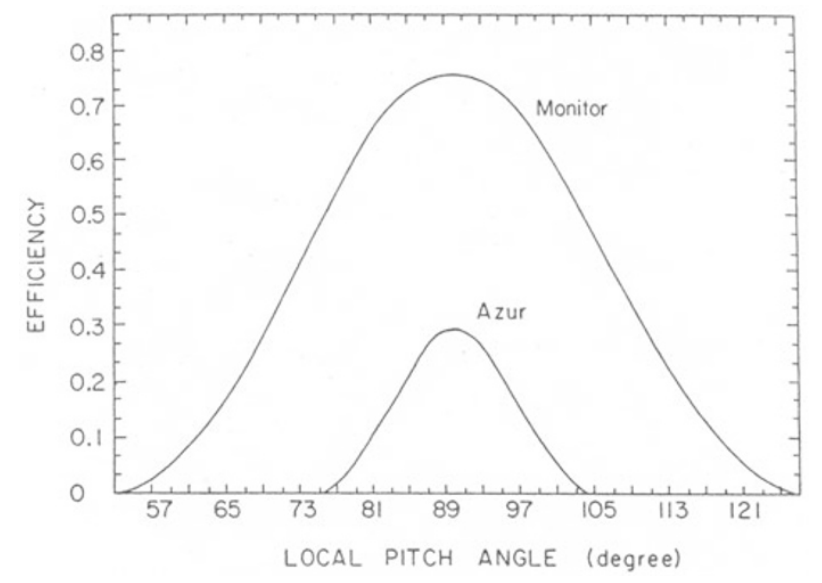

Fig. 12. Illustrates the response functions of the monitor telescope on board the ONR-602 Experiment and the Azur telescope on board the German Research Satellite.

In the dipole field, the peak efficiency was for $\alpha_{e}=90^{\circ}$. But in the real field geomagnetic equator, the response function peaks for $\alpha_{e}=90 \pm 7^{\circ}$. This demonstrates the influence of the magnetic field variation, a magnetospheric activity.

Regarding an application example to a specific instrument and observation data, the method developed was used to calculate the response functions of the monitor telescope in the ONR-602 Experiment and the German Research Satellite Azur. Figure 12 shows the sampling efficiency as a function of the pitch angles at the dipole equator. Azur telescope axis was within $90 \pm 5^{\circ}$ with the magnetic field at the equator. Its low opening angle accounts for its low values of response function.

As to the use in observational data, the detector count rate in the pitch angle range $\alpha_{1}$ to $\alpha_{2}$, and energy range $E_{1}$ to $E_{2}$ during a readout time $T$ is given by the integral over the incoming particle direction $\mathbf{r}$ of the product of particle flux $j(E, B, L, \alpha, \lambda, \phi, t, q)$ with the detector area $A$ exposed to the incident direction i.e.,

$$
\begin{aligned}
R= & \frac{1}{T} \int_{0}^{T} d t \int_{E_{1}}^{E_{2}} d E \int_{\Omega} d \omega \int_{A} d \mathbf{A} \cdot \mathbf{r}(\omega) \\
& \cdot j(E, B, L, \alpha, \lambda, \phi, t, q)
\end{aligned}
$$




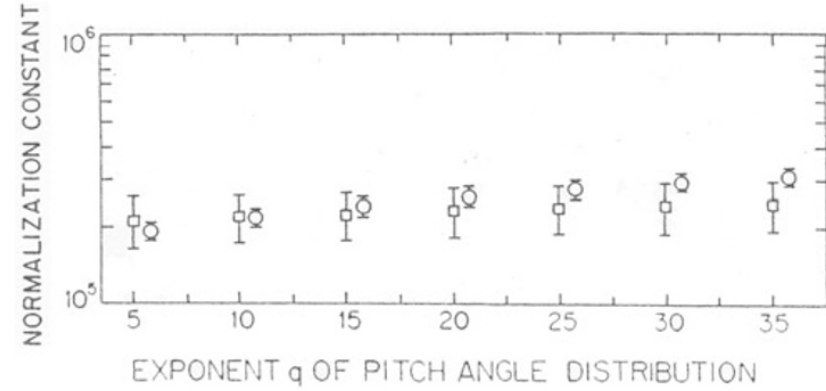

Fig. 13. Illustrates the comparison of the normalization constant $J_{n}(h, t)$ for different values of $q$, the pitch angle distribution function exponent. The circles represent $J_{n}(h, t)$ for the monitor telescope on board the ONR-602 Experiment. The squares represent the same values for German Research Satellite telescope Azur.

We assume that the most general expression for the flux has the form

$j(E, B, L, \alpha, \lambda, \phi, t, q)=J_{n}(B, L, \lambda, \phi, t, q) E^{-b} \sin ^{q} \alpha$

where $J_{n}$ is the normalization constant, which characterizes the actual particle population, $\sin ^{q} \alpha$ is the pitch angle distribution, and $E^{-b}$ is the energy spectrum. The counting rate can be written as

$$
\begin{aligned}
R= & J_{n}(B, L, \lambda, \phi, t, q) \frac{1}{T} \int_{0}^{T} d t \int_{E_{1}}^{E_{2}} E^{-b} d E \int_{\alpha_{1}}^{\alpha_{2}} d \alpha \\
& \cdot \int_{d \Omega} d \omega \int_{A} d \mathbf{A} \cdot \mathbf{r}(\omega) \cdot \sin ^{q} \alpha \\
= & J_{n} Q G
\end{aligned}
$$

where

$$
Q=\int_{E_{1}}^{E_{2}} E^{-b} d E
$$

and $G$ is given by Eq. (55) above, and can be written as

$$
G=A F
$$

where

$$
F=\sum_{j=1}^{m} F\left(\alpha_{j}\right) f\left(\alpha_{j}\right) \Delta \alpha
$$

and

$$
\Delta \alpha=\frac{\alpha_{2}-\alpha_{1}}{m}
$$

Then

$$
R=A F J_{n} \cdot Q
$$

The flux normalization constant is then given by

$$
J_{n}=\frac{R}{A F Q}
$$

$J_{n}$ depends on the exponent $q$ of the pitch angle distribution function and the epoch or time $t$ (Miah, 1988). Considering a range of values for $q$, we can study the variation of $J_{n}$ with time $t$. Since $J_{n}$ is representative of the particle population, comparison of $J_{n}$ 's for different epochs is basically a comparison of the particle population at different epochs. In Fig. 13, the open circles represent the $J_{n}(h, t)$ values for the monitor detector flux in ONR-602 Experiment observed at $277 \mathrm{~km}$ and the squares represent the same values for Azur for measurements at $450 \mathrm{~km}$. For clarity, squares and circles have been offset along the horizontal scale. Within the uncertainties, the fluxes measured by Moritz (1972) at $450 \mathrm{~km}$ and by the monitor detector at $277 \mathrm{~km}$ are indistinguishable. Altitude difference and altitude-dependent source depletion model can explain why the fluxes from the two measurements are indistinguishable (Miah, 1988).

\section{Conclusion}

Although the treatment given here is for the particle telescope used in the ONR-602 experiment, this geometrical method can be extended for application for the determination of the pitch angle sampling capability of a particle telescope of any orientation and for the parent satellite of any orbital inclination. A computer code can be generated to perform the rotations of axes, determination of the pitch angle ranges, and the evaluation of the efficiency function. The response function depends on the orientation of the magnetic field and as such the response functions calculated at the dipole magnetic field equator differs slightly from the response function calculated using any standard magnetic field model. Relating the sampling function to the count rate, it is possible to make absolute comparison of magnetospheric particle population pertaining to different epochs.

Acknowledgments. The work was partly supported at the Louisiana State University, Baton Rouge, LA by ONR under contract N-00014-83-K-0365. The work for the preparation of the manuscript was completed at the University of Arkansas at Pine Bluff. The United States Government is authorized to reproduce and distribute reprints for governmental purposes notwithstanding any copyright notation thereon.

\section{References}

Adel, M. M., Absolute flux comparison of magnetospheric particle, Can. Astronaut. Space J., 2007 (submitted).

Hovestadt, D., B. Hausler, and M. Scholer, Observation of energetic particles at very low altitudes near the geomagnetic equator, Phys. Rev. Lett., 28(20), 1340-1344, 1972.

Miah, M. A., Global Zones of Particle Precipitations, Ph.D. Thesis, Louisiana State University, p. 107, 1988.

Miah, M. A., The ONR-602 Experiment and investigation of particle precipitation near the equator, J. Geomag. Geoelectr., 43, 445-460, 1991a.

Miah, M. A., Observation of $\mathrm{Z} \geq 1$ particles below $300 \mathrm{~km}$ near the geomagnetic equator, J. Geomag. Geoelectr., 43, 461-475, $1991 \mathrm{~b}$.

Miah, M. A., Significant variation of proton population in the equatorial thermosphere, Adv. Space Res., 14(9), 229-232, 1994.

Miah, M. A., J. W. Mitchell, and J. P. Wefel, Magnetospheric particle detection efficiency of a conical telescope, Nucl. Instrum. Methods Phys. Res., A281, 622-627,1989.

Miah, M. A., K. Nagata, T. Kohno, H. Murakami, A. Nakamoto, N. Hasebe, J. Kikuchi, and T. Doke, Spatial and temporal features of 0.64$35 \mathrm{MeV}$ protons in the Space Station environment: EXOS-C observations, J. Geomag. Geoelectr., 44, 591-610, 1992.

Moritz, J., Energetic protons at low equatorial altitudes, Zeitschrift fur Geophysik, 38, 701-717, 1972.

M. M. Adel (e-mail: adelm@uapb.edu) 